\title{
Aarrso Autopercepção positiva da alimentação: um estudo de base populacional no extremo sul do Brasil $^{*}$ \\ doi: $10.5123 /$ S1679-49742019000300005
}

\author{
Positive self-perception of diet: a population-based study in the far south of Brazil \\ Autopercepción positiva de la alimentación: un estudio de base poblacional en el extremo \\ sur de Brasil
}

\author{
Karla Pereira Machado' 1 - (1) orcid.org/0000-0003-1765-1435 \\ Juliana dos Santos Vaz ${ }^{2}$ - (1) orcid.org/0000-0002-2880-767X \\ Raúl Andrés Mendoza-Sassi' - — orcid.org/0000-0002-4641-9056 \\ 'Universidade Federal do Rio Grande, Programa de Pós-Graduação em Saúde Pública, Rio Grande, RS, Brasil \\ ${ }^{2}$ Universidade Federal de Pelotas, Programa de Pós-Graduação em Nutrição e Alimentos, Pelotas, RS, Brasil
}

\section{Resumo}

Objetivo: analisar a prevalência de autopercepção positiva da alimentação e sua associação com fatores sociodemográficos, comportamentais, morbidades, autopercepção da saúde e do peso e cuidados com o peso corporal, no município de Rio Grande, RS, Brasil, em 2016. Métodos: estudo transversal realizado com adultos e idosos residentes no município. Foram aplicados 13 indicadores de alimentação saudável e autopercepção de alimentação (desfecho). Empregou-se regressão de Poisson. Resultados: a amostra foi de 1.243 indivíduos, dos quais $72,4 \%$ referiram autopercepção positiva da alimentação. Indicadores como consumo semanal de verduras ou legumes $\left(\mathrm{RP}=1,33-\mathrm{IC}_{95 \%} 1,22 ; 1,45\right)$ e frutas $\left(\mathrm{RP}=1,35-\mathrm{IC}_{95 \%}\right.$ $1,22 ; 1,48)$ foram positivamente associados ao desfecho. Não houve diferença significativa entre os sexos. Conclusão: a autopercepção de alimentação positiva se associa com indicadores de alimentação entre adultos e idosos, podendo ser útil em estudos epidemiológicos.

Palavras-chave: Consumo de Alimentos; Autorrelato; Inquéritos Epidemiológicos; Estudos Transversais. 


\section{Introdução}

O Guia Alimentar para a População Brasileira e os dez passos para uma alimentação saudável - documento oficial do Ministério da Saúde, dedica-se à abordagem e recomendação de uma alimentação adequada e saudável, configurando-se como instrumentos de extrema importância no sentido de se alcançar uma mudança de comportamento na população brasileira. ${ }^{1-3}$ Quando o nível de informação do indivíduo sobre saúde e nutrição é adequado e sua percepção individual é crítica, torna-se possível a adesão a um maior número de indicadores saudáveis, ${ }^{4,5}$ independentemente da renda. Esta, ou o preço dos alimentos, é um dos principais fatores de influência no comportamento saudável, e, por conseguinte, um quesito a ser avaliado na hora da escolha e compra de alimentos. ${ }^{6}$

A relação entre a autopercepção da qualidade da alimentação do indivíduo e a ocorrência de uma alimentação realmente saudável, contudo, é pouco explorada na literatura nacional e internacional. Os estudos existentes sobre o tema avaliaram esse desfecho, principalmente entre escolares e adolescentes, e identificaram uma relação direta entre autopercepção positiva da alimentação e hábitos alimentares mais adequados. ${ }^{1,2,4}$ Nas pesquisas sobre alimentação e nutrição, o hábito alimentar é avaliado mediante aplicação de um questionário de frequência alimentar ou a partir de inquéritos padronizados que, geralmente, demandam longo tempo de realização. ${ }^{7,8}$ Trata-se de instrumentos pouco práticos em situações nas quais o contato entre o profissional de saúde e o indivíduo é breve. A importância de propor uma questão sobre a autopercepção, capaz de identificar o hábito alimentar dos indivíduos ${ }^{4,9}$ e o risco representado pelo hábito de uma alimentação não saudável, deve se refletir na indicação de grupos-alvo para possíveis intervenções. ${ }^{10}$

\section{Quando o nível de informação do indivíduo sobre saúde e nutrição é adequado e sua percepção individual é crítica, torna-se possível a adesão a um maior número de indicadores saudáveis, independentemente da renda.}

0 objetivo do presente artigo foi analisar a prevalência de autopercepção positiva da alimentação e sua associação com fatores sociodemográficos, comportamentais, morbidades, autopercepção de saúde/peso e cuidados com o peso corporal no município de Rio Grande, estado do Rio Grande do Sul, Brasil, em 2016.

\section{Métodos}

Trata-se de um estudo transversal de base populacional realizado no sul do Rio Grande do Sul, parte de uma pesquisa maior, intitulada 'Saúde da População Riograndina, RS', ${ }^{11}$ cuja finalidade foi investigar diversos aspectos característicos do perfil de saúde da população de Rio Grande. Este município, situado no extremo sul do estado, possuía aproximadamente 200 mil habitantes ${ }^{12} \mathrm{e}$ apresentava um índice de desenvolvimento humano (IDH) de 0,744 no ano de 2010. ${ }^{13}$ Em 2015, a atividade portuária se mantinha como sua principal base econômica, e o produto interno bruto (PIB) do município se aproximava dos $\mathbf{R} \$ 35.000,00$ per capita/ano.

A população-alvo do estudo foi composta por indivíduos com 20 anos ou mais de idade, residentes na área urbana de Rio Grande. Foram excluídos aqueles que estavam institucionalizados em asilos, hospitais e presídios, ou que apresentavam alguma incapacidade física e/ou mental para responder ao questionário.

0 cálculo amostral foi realizado utilizando-se, como parâmetro, uma prevalência do desfecho estudado de $51 \%,{ }^{2,10,14}$ nível de confiança de $95 \%$ e erro aceitável de 3 pontos percentuais. Para os fatores associados, considerou-se uma razão de prevalência $(\mathrm{RP})^{13}$ de 1,5 , nível de confiança de $95 \%$, poder de $80 \%$, frequência de expostos entre 35 e $50 \%$, além de efeito de delineamento de $1,5,10 \%$ para perdas e $15 \%$ para controle de fatores de confusão. Afinal, o tamanho da amostra totalizou 1.152 indivíduos. Os cálculos foram realizados pelo programa Epi Info 7.0 (Centers for Disease Control and Prevention, Atlanta, Estados Unidos). Como esta pesquisa integrava um estudo maior, com diferentes desfechos, decidiu-se utilizar o maior tamanho de amostra calculado $(n=1.423)$, tornando o estudo representativo para as diferentes variáveis independentes investigadas, sendo considerado um poder de $90 \%$ para o n de 1.243 .

Para garantir a representatividade da amostra, o processo de amostragem ocorreu em dois estágios: setor censitário e domicílio, de acordo com os dados do Censo Demográfico 2010. ${ }^{12}$ Inicialmente, foram 
identificados os 293 setores censitários da zona urbana de Rio Grande, e destes, feita a seleção sistemática sobre todos os domicílios urbanos (77.835), colocados em ordem decrescente conforme a renda média mensal do chefe de família; após a seleção aleatória do primeiro domicilio, seguiu-se a seleção sistemática, mediante "pulo" de 1.080 domicílios, selecionando-se, assim, os 72 setores censitários.

Logo, promoveu-se a seleção aleatória para determinar os domicílios investigados, chegou-se a 711 residências, considerando-se um "pulo" de 32 residências, e uma média esperada de 2,08 indivíduos por domicílio. ${ }^{12}$ A seleção dos setores e dos domicílios foi realizada mediante probabilidade proporcional à população e ao número de domicílios por setor.

A coleta de dados aconteceu entre abril e julho de 2016, realizada por nove entrevistadoras previamente treinadas. As entrevistas foram feitas no domicílio selecionado, com todos os moradores elegíveis, seguindo o roteiro de um questionário estruturado, padronizado e previamente testado, composto por questões sociodemográficas e de saúde geral, pré-codificadas.

0 desfecho do estudo, a autopercepção positiva da alimentação, foi investigado pela questão "Como o Sr. (a) considera a sua alimentação?" e com as seguintes alternativas de resposta para ela: 'muito boa', 'boa', 'regular', 'ruim' ou 'muito ruim'. Para fins de análise, essa variável foi dicotomizada entre positiva (muito boal boa) e negativa (regular/ruim/muito ruim).

Foram utilizados 13 indicadores de alimentação saudável, coletados sobre uma semana habitual para os indivíduos, estabelecidos conforme as recomendações do Guia Alimentar para a População Brasileira. A publicação, reformulada em $2014,{ }^{3}$ orienta a preferência pelo consumo de alimentos in natura ou minimamente processados, ao invés de alimentos ultraprocessados, de forma a constituir uma dieta com refeições saudáveis. ${ }^{3}$ Eis os indicadores utilizados:

I) realização do café da manhãa

II) realização do almoço;

III) realização do lanche ou café da tarde;

IV) realização do jantar;

V) realização do lanche ou café antes de dormir;

VI) hábito de comer gordura aparente da carne vermelha e/ou comer a pele do frango; $\mathrm{e}$

VII) sal adicional.

Os indicadores I a VII foram coletados de forma dicotômica (sim/não); para I a V, considerou-se con- sumo apropriado quando a resposta era 'sim', e para VI e VII, quando a resposta era 'não'.

Outros seis indicadores relativos à frequência de consumo foram coletados:

VIII) número de dias na semana em que costuma comer leguminosas;

IX) número de dias na semana em que costuma comer verduras ou legumes;

X) número de dias na semana em que costuma comer frutas;

XI) número de dias na semana em que costuma comer peixe;

XII) número de dias na semana em que costuma comer alimentos com açúcar como ingrediente - sorvetes, chocolates, bolos, biscoitos ou doces -; e

XIII) número de dias na semana em que costuma tomar refrigerante ou suco artificial.

Tais indicadores foram coletados de forma politômica: (a) nunca/quase nunca; (b) 1 a 2 dias por semana; (c) 3 a 4 dias por semana; (d) 5 a 6 dias por semana; (e) todos os dias (inclusive sábado e domingo). Posteriormente, para fins de análise, os indicadores foram dicotomizados com base nas orientações do Guia Alimentar para a População Brasileira, adaptado. ${ }^{3}$ Para os indicadores VIII a XI, o consumo foi considerado adequado quando a frequência foi igual ou superior a 3 vezes por semana; já para os indicadores XII e XIII, considerou-se consumo adequado as frequências 'nunca/quase nunca' e ' 1 a 2 dias por semana'.

As variáveis independentes incluídas foram:

a) Sociodemográficas

- sexo (masculino; feminino);

-idade (em anos: 20-29; 30-39; 40-49; 50-59; 60 ou mais);

- raça/cor da pele autorreferida (branca; preta/parda/ amarela/indígena);

- estado civil (casado; solteiro; divorciado/separado/viúvo); - escolaridade (em anos de estudo: 0-4; 5-8; 9-11; 12 ou mais); e

- renda (em quartis: $1^{\circ}$ quartil [mais pobre], $R \$ 293,3$ a $\mathbf{R}$ \$ 520,0; $2^{\circ}$ quartil, $\mathrm{R} \$ 750,0$ a $\mathbf{R}$ \$ 905,5; $3^{\circ}$ quartil, $\mathrm{R} \$ 1.166,7$ a $\mathrm{R} \$ 1.500,0 ; \mathrm{e} 4^{\circ}$ quartil [mais rico], $\mathrm{R} \$ 2.200,0$ a R $\$ 4.400,0)$, considerando-se o salário mínimo de $R \$ 880,00$ reais no ano de 2016.

b) Comportamentais (opções de resposta: 'sim' ou 'não') - tabagismo (não fumante; ex-fumante/fumante); - consumo de bebida alcoólica (não bebe; bebe menos de uma vez por semana/número de vezes em que bebe, por semana); 
- atividade física no lazer, medida pela seção 'Lazer' do Questionário Internacional de Atividade Física (IPAQ-SHORT), que define a condição de ativo como 0 indivíduo que mantém $\geq 150$ minutos de atividade física por semana ${ }^{15}$

c) Índice de massa corporal (IMC) (baixo peso; eutrópico; sobrepeso; obeso);

d) Morbidades autorreferidas (presença ou ausência) de diabetes; hipertensão; depressão variável coletada pelo instrumento Patient Health Questionnaire (PHQ-9), que define a condição de depressão $\geq 9 ;{ }^{16}$

e) Segurança alimentar, medida pela Escala Brasileira de Insegurança Alimentar (EBIA) (segurança alimentar; insegurança alimentar leve; insegurança alimentar moderada/grave) $;{ }^{17}$

f) Autopercepção de saúde (excelente/muito boa; boa; regular/ruim)

g) Autopercepção do peso corporal (normal; muito magro/magro/um pouco magro; muito gordo/ gordo/um pouco gordo); e

h) Cuidados com o peso corporal, variável coletada mediante a proposição da questão " $O(a) S r$ (a) está fazendo alguma coisa para perder peso ou ganhar massa muscular?" e suas opções de resposta 'sim' ou 'não'.

Todos os questionários foram revisados e duplamente digitados por diferentes digitadores, utilizando-se 0 software EpiData $\AA^{3.1}$ (EpiData Association, Dinamarca). 0 controle de qualidade foi realizado com a repetição parcial de $10,5 \%$ das entrevistas com perguntas-chave selecionadas, para avaliar a confiabilidade do instrumento. 0 valor médio do coeficiente de concordância de Kappa entre as perguntas foi de 0,80 (variação de 0,27 [número de vezes na semana em que caminhava no tempo livre] a 0,93 [existência de telefone fixo no domicílio]), revelando concordância substancial. ${ }^{18}$

Primeiramente, foram calculadas as frequências absoluta e relativa das cinco categorias da escala de Likert contidas no desfecho (autopercepção positiva da alimentação), e a prevalência e o intervalo de confiança de $95 \%\left(\mathrm{IC}_{95 \%}\right)$ do desfecho dicotomizado.

Para estudar o impacto das variáveis independentes sobre o desfecho, foi utilizada a regressão de Poisson, levando-se em consideração o efeito do delineamento do estudo. Foram calculadas as razões de prevalência (RP) e seus IC ${ }_{95 \%}$. Empregou-se um modelo hierárquico de quatro níveis. 0 primeiro nível foi composto pelas variáveis demográficas e socioeconômicas, e o segun- do nível, pelas variáveis comportamentais. 0 terceiro nível contemplou variáveis referentes a morbidade e segurança alimentar, e o quarto nível, as variáveis de autopercepção de saúde, autopercepção e cuidados com o peso corporal. As exposições de interesse foram ajustadas para variáveis de mesmo nível e de níveis superiores, permanecendo no modelo apenas aquelas com $\mathrm{p} \leq 0,20$. 0 teste estatístico empregado foi o teste de Wald.

Logo, foi estimada a RP e o IC ${ }_{95 \%}$ de cada um dos indicadores de consumo alimentar. Finalmente, a aplicação do teste de proporções permitiu a análise das diferenças entre os indicadores de consumo alimentar e o desfecho 'autopercepção saudável', estratificado por sexo.

Para a análise dos dados, foi utilizado o programa Stata ${ }^{\circledR}$ versão 14.0 (StataCorp/College Station, Estados Unidos). 0 nível de significância menor que 5\% foi adotado para testes bicaudais, e 0 efeito de delineamento amostral foi levado em consideração em todas as análises, utilizando-se o comando survey (svy).

0 protocolo da pesquisa foi submetido e aprovado pelo Comitê de Ética em Pesquisa na Área da Saúde (CEPAS) da Universidade Federal do Rio Grande (FURG), sob o número 20/2016. Os princípios éticos foram assegurados, foi apresentado aos entrevistados 0 Termo de Consentimento Livre e Esclarecido, assinado por todos, de maneira a garantir o direito de não participação na pesquisa e 0 anonimato dos participantes.

\section{Resultados}

Foram respondidos 1.243 questionários $(8,9 \%$ de perdas e recusas). 0 desfecho do estudo foi respondido por 1.240 indivíduos. Mais da metade era do sexo feminino $(56,4 \%)$, a maioria autodeclarou-se de raça/ cor da pele branca $(83,1 \%)$ e a média de idade foi de 47 anos (desvio-padrão [DP]: $\pm 16,6$ anos). Cerca de 57\% tinham mais de 9 anos de estudo, e a mediana de renda foi de $\mathrm{R} \$ 1.000,00$ (P25: R\$ 625,00-P75; $\mathrm{R} \$ 1.764,00)$. A maioria dos indivíduos não realizava atividade física de lazer $(77,5 \%)$ e a insegurança alimentar foi encontrada em 34,5\% dos domicílios (Tabela 1).

A frequência da autopercepção alimentar foi 16,9\% muito boa; $55,5 \%$ boa; $23,2 \%$ regular; 3,4\% ruim; e $1,0 \%$ muito ruim. Quando dicotomizada, a prevalência de autopercepção positiva da alimentação foi de $72,4 \%$ $\left(\mathrm{IC}_{95 \%} 69,9 ; 74,9\right)$. 
Tabela 1 - Descrição da amostra de adultos e idosos ( $n=1.243)$, Rio Grande, Rio Grande do Sul, 2016

\begin{tabular}{|c|c|c|}
\hline \multirow{2}{*}{ Variáveis } & \multicolumn{2}{|c|}{ Amostra geral } \\
\hline & N & $\%$ \\
\hline \multicolumn{3}{|l|}{ Sexo } \\
\hline Masculino & 542 & 43,6 \\
\hline Feminino & 701 & 56,4 \\
\hline \multicolumn{3}{|l|}{ Idade (em anos) } \\
\hline $20-29$ & 226 & 18,2 \\
\hline $30-39$ & 225 & 18,1 \\
\hline $40-49$ & 240 & 19,3 \\
\hline $50-59$ & 237 & 19,1 \\
\hline$\geq 60$ & 315 & 25,3 \\
\hline \multicolumn{3}{|l|}{ Raça/cor da pele ( $n=1.241)$} \\
\hline Branca & 1.301 & 83,1 \\
\hline Preta/parda/amarela/indígena & 210 & 16,9 \\
\hline \multicolumn{3}{|l|}{ Estado civil } \\
\hline Casado & 476 & 38,3 \\
\hline Solteiro & 545 & 43,8 \\
\hline Divorciado/separado/viúvo & 222 & 17,9 \\
\hline \multicolumn{3}{|c|}{ Escolaridade (em anos de estudo) $(n=1.241)$} \\
\hline $0-4$ & 190 & 15,3 \\
\hline $5-8$ & 343 & 27,6 \\
\hline $9-11$ & 367 & 29,6 \\
\hline$\geq 12$ & 341 & 27,5 \\
\hline \multicolumn{3}{|l|}{ Renda em quartis ( $n=1.123$ ) } \\
\hline $1^{\circ}$ quartil (mais pobre) & 284 & 25,3 \\
\hline $2^{\circ}$ quartil & 306 & 27,2 \\
\hline $3^{\circ}$ quartil & 255 & 22,7 \\
\hline $4^{0}$ quartil (mais rico) & 278 & 24,8 \\
\hline \multicolumn{3}{|l|}{ Tabagismo } \\
\hline Não & 1.003 & 81,5 \\
\hline Sim & 230 & 18,5 \\
\hline \multicolumn{3}{|c|}{ Consumo de bebida alcoólica ( $n=1.242)$} \\
\hline Não & 896 & 72,1 \\
\hline Sim & 346 & 27,9 \\
\hline \multicolumn{3}{|c|}{ Atividade física no lazer $(\mathrm{n}=1.233)$} \\
\hline Insuficientemente ativo & 956 & 77,5 \\
\hline Suficientemente ativo & 277 & 22,5 \\
\hline
\end{tabular}


Tabela 1 - Descrição da amostra de adultos e idosos (n=1.243), Rio Grande, Rio Grande do Sul, 2016

\begin{tabular}{|c|c|c|}
\hline \multirow{2}{*}{ Variáveis } & \multicolumn{2}{|c|}{ Amostra geral } \\
\hline & $\mathbf{N}$ & $\%$ \\
\hline \multicolumn{3}{|l|}{ Índice de massa corporal (IMC) (n=1.189) } \\
\hline Baixo peso & 14 & 1,2 \\
\hline Eutrófico & 431 & 36,2 \\
\hline Sobrepeso & 458 & 38,5 \\
\hline Obeso & 286 & 24,1 \\
\hline \multicolumn{3}{|l|}{ Diabetes (autorreferida) $(n=1.239)$} \\
\hline Não & 1.149 & 92,7 \\
\hline Sim & 90 & 7,3 \\
\hline \multicolumn{3}{|l|}{ Hipertensão (autorreferida) $(\mathrm{n}=1.242$ ) } \\
\hline Não & 879 & 70,8 \\
\hline Sim & 363 & 29,2 \\
\hline \multicolumn{3}{|l|}{ 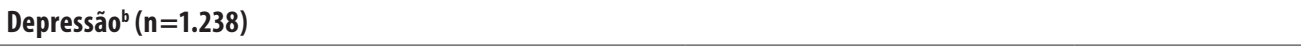 } \\
\hline Não & 893 & 72,1 \\
\hline Sim & 345 & 27,9 \\
\hline \multicolumn{3}{|c|}{ Autopercepção de alimentação saudável ( $n=1.240)$} \\
\hline Negativa & 342 & 27,6 \\
\hline Positiva & 898 & 72,4 \\
\hline \multicolumn{3}{|l|}{ Insegurança alimentarc $(n=1.224)$} \\
\hline Segurança alimentar & 802 & 65,5 \\
\hline Insegurança alimentar leve & 330 & 27,0 \\
\hline Insegurança alimentar moderada/grave & 92 & 7,5 \\
\hline \multicolumn{3}{|l|}{ Autopercepção de saúde } \\
\hline Excelente/muito boa & 263 & 21,2 \\
\hline Boa & 560 & 45,0 \\
\hline Regular/ruim & 420 & 33,8 \\
\hline \multicolumn{3}{|l|}{ Autopercepção do peso corporal $(n=1.242)$} \\
\hline Normal & 123 & 9,9 \\
\hline Muito magro/magro/um pouco magro & 563 & 45,3 \\
\hline Muito gordo/gordo/um pouco gordo & 556 & 44,8 \\
\hline \multicolumn{3}{|l|}{ Cuidados com o peso corporal } \\
\hline Não & 973 & 78,3 \\
\hline Sim & 270 & 21,7 \\
\hline Total & 1.243 & 100,0 \\
\hline
\end{tabular}

As RP brutas e ajustadas para o desfecho se encontram na Tabela 2. Na análise ajustada, as mulheres apresentaram menor prevalência do desfecho, em comparação aos homens $\left(\mathrm{RP}=0,94-\mathrm{IC}_{95 \%} 0,80 ; 1,00\right)$. os indivíduos na idade de 60 anos ou mais apresentaram
1,28 vez maior probabilidade do desfecho, comparados àqueles com 20-29 anos; a probabilidade também foi maior para os situados no quartil de renda mais elevada ( $\left.\mathrm{RP}=1,17-\mathrm{IC}_{95 \%} 1,04 ; 1,32\right)$, em relação aos do quartil mais pobre. No segundo nível, os indivíduos que 
Tabela 2 - Prevalência e análise bruta e ajustada de fatores associados à autopercepção positiva da alimentação em adultos e idosos ( $n=1.240)$, Rio Grande, Rio Grande do Sul, 2016

\begin{tabular}{|c|c|c|c|c|c|}
\hline \multirow[b]{2}{*}{ Variáveis } & \multirow{2}{*}{$\begin{array}{l}\text { Prevalência de autopercepção } \\
\text { positiva da alimentação } \\
(n=898) \\
\mathrm{N}(\%)\end{array}$} & \multicolumn{2}{|c|}{ Análise bruta } & \multicolumn{2}{|c|}{ Análise ajustada } \\
\hline & & $\operatorname{RP}^{\mathrm{a}}\left(\mathrm{IC}_{95 \%}{ }^{\mathrm{b}}\right)$ & $\mathbf{p}^{c}$ & $\operatorname{RP}^{\mathrm{a}}\left(\mathrm{IC}_{95 \%}{ }^{\mathrm{b}}\right)$ & $\mathbf{p}^{c}$ \\
\hline \multicolumn{6}{|c|}{$1^{\circ}$ nível } \\
\hline Sexo & & & 0,297 & & 0,071 \\
\hline Masculino & $400(73,9)$ & 1,00 & & 1,00 & \\
\hline Feminino & $498(71,2)$ & $0,97(0,90 ; 1,03)$ & & $0,94(0,88 ; 1,00)$ & \\
\hline Idade (em anos) & & & $<0,001$ & & $<0,001$ \\
\hline $20-29$ & $142(62,8)$ & 1,00 & & 1,00 & \\
\hline $30-39$ & $140(62,2)$ & $0,98(0,84 ; 1,14)$ & & $0,93(0,80 ; 1,08)$ & \\
\hline $40-49$ & $163(67,9)$ & $1,08(0,93 ; 1,25)$ & & $1,06(0,92 ; 1,22)$ & \\
\hline $50-59$ & $190(80,2)$ & $1,26(1,12 ; 1,44)$ & & $1,23(1,09 ; 1,39)$ & \\
\hline$\geq 60$ & $263(84,3)$ & $1,33(1,17 ; 1,51)$ & & $1,28(1,13 ; 1,44)$ & \\
\hline Raça/cor da pele $(n=896)$ & & & \multicolumn{2}{|l|}{0,594} & 0,249 \\
\hline Branca & $739(71,9)$ & 1,00 & & 1,00 & \\
\hline Preta/parda/amarela/indígena & $157(74,8)$ & $1,03(0,93 ; 1,14)$ & \multirow{2}{*}{\multicolumn{2}{|c|}{$1,05(0,95 ; 1,16)$}} & \\
\hline Estado civil & & & & & 0,641 \\
\hline Casado & $360(75,8)$ & 1,00 & & 1,00 & \\
\hline Solteiro & $367(67,6)$ & $0,90(0,82 ; 0,98)$ & & $0,99(0,89 ; 1,09)$ & \\
\hline Divorciado/separado/viúvo & $171(77,0)$ & $1,01(0,93 ; 1,11)$ & & $0,96(0,87 ; 1,05)$ & \\
\hline Escolaridade (em anos de estudo) $(n=897)$ & & & \multicolumn{2}{|c|}{$0,081^{\mathrm{d}}$} & $0,395^{\mathrm{d}}$ \\
\hline $0-4$ & $144(76,6)$ & 1,00 & & 1,00 & \\
\hline $5-8$ & $257(74,9)$ & $0,98(0,88 ; 1,10)$ & & $0,99(0,88 ; 1,11)$ & \\
\hline $9-11$ & $260(71,0)$ & $0,93(0,82 ; 1,06)$ & & $0,96(0,83 ; 1,11)$ & \\
\hline$\geq 12$ & $236(69,2)$ & $0,92(0,81 ; 1,03)$ & & $0,95(0,82 ; 1,11)$ & \\
\hline Renda em quartis (mediana; P25/P75 em R\$) $(n=810)$ & & & $0,002^{\mathrm{d}}$ & & $0,017^{\mathrm{d}}$ \\
\hline $1^{0}$ quartil mais pobre $(\mathrm{R} \$ 293,3$ a $\mathrm{R} \$ 520,0)$ & $183(64,4)$ & 1,00 & & 1,00 & \\
\hline $2^{\circ}$ quartil $(\mathrm{R} \$ 750,0$ a $\mathrm{R} \$ 905,5)$ & $219(72,3)$ & $1,13(1,01 ; 1,25)$ & & $1,09(0,98 ; 1,22)$ & \\
\hline $3^{\circ}$ quartil ( $R \$ 1.166,7$ a $\left.R \$ 1.500,0\right)$ & $187(73,3)$ & $1,14(1,01 ; 1,28)$ & & $1,07(0,95 ; 1,20)$ & \\
\hline $4^{0}$ quartil mais rico ( $R \$ 2.200,0$ a $\left.R \$ 4.400,0\right)$ & $221(79,5)$ & $1,24(1,10 ; 1,40)$ & & $1,17(1,04 ; 1,32)$ & \\
\hline \multicolumn{6}{|c|}{$2^{\circ}$ nível } \\
\hline Tabagismo atual & & & 0,205 & & 0,398 \\
\hline Não & $740(73,2)$ & 1,00 & & 1,00 & \\
\hline Sim & $158(69,0)$ & $0,94(0,86 ; 1,04)$ & & $0,95(0,84 ; 1,07)$ & \\
\hline Consumo de bebida alcoólica $(n=897)$ & & & 0,564 & & 0,927 \\
\hline Não & $643(71,0)$ & 1,00 & & 1,00 & \\
\hline Sim & $254(73,6)$ & $1,03(0,95 ; 1,11)$ & & $1,00(0,91 ; 1,09)$ & \\
\hline Atividade física no lazer ${ }^{f}(n=893)$ & & & $<0,001$ & & $<0,001$ \\
\hline Insuficientemente ativo & $667(69,8)$ & 1,00 & & 1,00 & \\
\hline Suficientemente ativo & $226(81,6)$ & $0,86(0,80 ; 0,92)$ & & $1,17(1,08 ; 1,26)$ & \\
\hline Índice de massa corporal (IMC) $(n=854)$ & & & $0,009^{d}$ & & $0,015^{\mathrm{d}}$ \\
\hline Baixo peso & $11(78,6)$ & 1,00 & & 1,00 & \\
\hline Eutrófico & $325(75,6)$ & $0,96(0,74 ; 1,25)$ & & $0,90(0,69 ; 1,17)$ & \\
\hline Sobrepeso & $329(71,8)$ & $0,91(0,71 ; 1,17)$ & & $0,86(0,67 ; 1,10)$ & \\
\hline Obeso & $189(66,1)$ & $0,85(0,65 ; 1,09)$ & & $0,80(0,62 ; 1,05)$ & \\
\hline
\end{tabular}

a) RP: razão de prevalência.

b) $\mathrm{IC}_{95 \%}$ : intervalo de confiança de $95 \%$.

c) Valor-p obtido pelo teste de Wald de heterogeneidade.

d) Valor-p obtido pelo teste de Wald para tendência linea

e) Considerando salário mínimo brasileiro em 2016 de $R \$ 880,00$.

f) Segundo a sessão 'Lazer' do Questionário Internacional de Atividade Física (IPAQ-SHORT), que define como ativo o indivíduo que mantém $\geq 150$ minutos de atividade física por semana.

g) Segundo 0 instrumento Patient Health Questionnaire (PHQ-9), que define a condição de depressão $\geq 9$.

h) Escala Brasileira de Insegurança Alimentar (EBIA).

Nota: Foi considerado 0 efeito de delineamento amostral. 
Tabela 2 - Prevalência e análise bruta e ajustada de fatores associados à autopercepção positiva da alimentação em adultos e idosos ( $n=1.240$ ), Rio Grande, Rio Grande do Sul, 2016

\begin{tabular}{|c|c|c|c|c|c|}
\hline \multirow[b]{2}{*}{ Variáveis } & \multirow{2}{*}{$\begin{array}{l}\text { Prevalência de autopercepção } \\
\text { positiva da alimentação } \\
(n=898) \\
N(\%)\end{array}$} & \multicolumn{2}{|c|}{ Análise bruta } & \multicolumn{2}{|c|}{ Análise ajustada } \\
\hline & & $\operatorname{RP}^{\mathrm{a}}\left(\mathrm{IC}_{95 \%}{ }^{\mathrm{b}}\right)$ & $\mathbf{p}^{c}$ & $\operatorname{RP}^{a}\left(\mathrm{IC}_{95 \%}{ }^{b}\right)$ & $\mathbf{p}^{c}$ \\
\hline \multicolumn{6}{|c|}{$3^{\circ}$ nível } \\
\hline Diabetes (autorreferida) $(n=895)$ & 0,972 & \multicolumn{3}{|c|}{0,761} & 0,071 \\
\hline Não & $829(72,3)$ & 1,00 & & 1,00 & \\
\hline Sim & $66(73,3)$ & $1,00(0,88 ; 1,14)$ & \multirow{2}{*}{\multicolumn{2}{|c|}{$0,98(0,84 ; 1,14)$}} & \\
\hline Hipertensão (autorreferida) & 0,791 & & & & $<0,001$ \\
\hline Não & $637(72,6)$ & 1,00 & \multicolumn{3}{|c|}{1,00} \\
\hline Sim & $261(72,1)$ & $0,99(0,92 ; 1,07)$ & \multicolumn{3}{|c|}{$0,93(0,85 ; 1,01)$} \\
\hline Depressãog $^{g}(n=896)$ & $<0,001$ & & \multicolumn{2}{|c|}{$<0,001$} & 0,249 \\
\hline Não & $697(78,1)$ & 1,00 & \multicolumn{3}{|c|}{1,00} \\
\hline Sim & $199(57,7)$ & $0,74(0,68 ; 0,81)$ & \multicolumn{3}{|c|}{$0,78(0,81 ; 0,95)$} \\
\hline Insegurança alimentar $(n=884)$ & $<0,001$ & & \multicolumn{2}{|l|}{0,006} & 0,641 \\
\hline Segurança alimentar & $604(75,5)$ & 1,00 & & 1,00 & \\
\hline Insegurança alimentar leve & $214(64,9)$ & $0,86(0,80 ; 092)$ & & $0,88(0,81 ; 0,95)$ & \\
\hline Insegurança alimentar moderada/grave & $66(71,7)$ & $0,95(0,81 ; 1,12)$ & & $1,01(0,85 ; 1,22)$ & \\
\hline \multicolumn{6}{|c|}{$4^{0}$ nível } \\
\hline Autopercepção de saúde & $<0,001 \mathrm{~d}$ & \multicolumn{3}{|c|}{$0,001^{d}$} & 0,398 \\
\hline Excelente/muito boa & $210(79,9)$ & 1,00 & & 1,00 & \\
\hline Boa & $439(78,4)$ & $0,98(0,91 ; 1,06)$ & & $0,98(0,89 ; 1,07)$ & \\
\hline Regular/ruim & $249(59,7)$ & $0,74(0,66 ; 0,83)$ & & $0,75(0,66 ; 0,86)$ & \\
\hline Cuidados com o peso corporal & $<0,001$ & & \multicolumn{2}{|l|}{0,030} & 0,927 \\
\hline Não & $682(70,3)$ & 1,00 & & 1,00 & \\
\hline Sim & $216(80,0)$ & $1,15(1,07 ; 1,23)$ & & $1,10(1,01 ; 1,21)$ & \\
\hline Autopercepção do peso corporal $(n=897)$ & $<0,001$ & & \multicolumn{2}{|c|}{$<0,001$} & $<0,001$ \\
\hline Eutrófico & $89(73,0)$ & 1,00 & & 1,00 & \\
\hline Magro (muito magro/um pouco magro/magro) & $452(80,4)$ & $1,09(0,97 ; 1,23)$ & & $0,99(0,88 ; 1,14)$ & \\
\hline Gordo (muito gordo/um pouco gordo/gordo) & $356(64,1)$ & $0,88(0,77 ; 1,00)$ & & $0,80(0,69 ; 0,95)$ & \\
\hline
\end{tabular}

a) RP: razão de prevalência.

b) $\mathcal{I}_{95 \%}$ : intervalo de confiança de $95 \%$.

c) Valor-p obtido pelo teste de Wald de heterogeneidade.

d) Valor-p obtido pelo teste de Wald para tendência linear.

e) Considerando salário mínimo brasileiro em 2016 de R\$ 880,00

f) Segundo a sessão 'Lazer' do Questionário Internacional de Atividade Física (IPAQ-SHORT), que define como ativo o indivíduo que mantém $\geq 150$ minutos de atividade física por semana.

g) Segundo o instrumento Patient Health Questionnaire (PHQ-9), que define a condição de depressão $\geq 9$.

h) Escala Brasileira de Insegurança Alimentar (EBIA).

Nota: Foi considerado o efeito de delineamento amostral.

realizavam 150 minutos ou mais de atividade física no lazer apresentaram um aumento no desfecho $(\mathrm{RP}=1,17$ - $\left.\mathrm{IC}_{95 \%} 1,08 ; 1,26\right)$. No terceiro nível, os indivíduos que relataram morbidades como diabetes $\left(\mathrm{RP}=0,98-\mathrm{IC}_{95 \%}\right.$ $0,84 ; 1,14)$ e depressão $\left(\mathrm{RP}=0,78-\mathrm{IC}_{95 \%} 0,81 ; 0,95\right)$ tiveram uma probabilidade menor de apresentar autopercepção positiva da alimentação. Em relação à insegurança alimentar, os indivíduos que apresentaram insegurança leve tiveram menor probabilidade do desfecho $\left(\mathrm{RP}=0,88-\mathrm{IC}_{95 \%}, 0,81 ; 0,95\right)$.

No quarto nível, observou-se que aqueles cuja percepção da própria saúde era regular ou ruim apresentaram menores prevalências de percepção positiva da alimentação
$\left(\mathrm{RP}=0,75-\mathrm{IC}_{95 \%} 0,66 ; 0,86\right)(\mathrm{p}=0,001)$, na comparação com quem relatou autopercepção de saúde excelente ou muito boa. Os indivíduos que referiram cuidado com 0 peso corporal tiveram maior probabilidade de perceber sua alimentação como positiva $\left(\mathrm{RP}=1,10-\mathrm{IC}_{95 \%} 1,01 ; 1,21\right)$.

Dos 13 indicadores que compõem a alimentação adequada, dez se mostraram significativamente associados com o desfecho (Tabela 3). A proporção de indivíduos com indicadores de alimentação saudável e autopercepção positiva da alimentação foram superiores a 70,0\%. Não houve diferença nas prevalências dos indicadores de alimentação saudável entre homens e mulheres (Tabela 4). 
Tabela 3 - Associação entre autopercepção positiva da alimentação e indicadores de alimentação saudável em adultos e idosos ( $n=1.240)$, Rio Grande, Rio Grande do Sul, 2016

\begin{tabular}{|c|c|c|}
\hline \multirow{2}{*}{ Variáveis } & \multicolumn{2}{|c|}{ Autopercepção positiva da alimentação } \\
\hline & $\mathrm{RPa}^{\mathrm{a}}$ & $I_{95 \%}{ }^{b}$ \\
\hline \multicolumn{3}{|c|}{ Realização do café da manhã } \\
\hline Não & 1,00 & - \\
\hline Sim & 1,46 & 1,$22 ; 1,75$ \\
\hline \multicolumn{3}{|c|}{ Realização do almoço } \\
\hline Não & 1,00 & - \\
\hline Sim & 1,77 & 1,$00 ; 3,13$ \\
\hline \multicolumn{3}{|c|}{ Realização do lanche ou café da tarde } \\
\hline Não & 1,00 & - \\
\hline Sim & 1,16 & 1,$05 ; 1,30$ \\
\hline \multicolumn{3}{|c|}{ Realização do jantar } \\
\hline Não & 1,00 & - \\
\hline Sim & 0,95 & 0,$84 ; 1,07$ \\
\hline \multicolumn{3}{|c|}{ Realização do lanche ou café antes de dormir } \\
\hline Não & 1,00 & - \\
\hline Sim & 1,19 & 1,$11 ; 1,27$ \\
\hline \multicolumn{3}{|c|}{ Consumo de leguminosas } \\
\hline Inadequado & 1,00 & - \\
\hline Adequado & 1,18 & 1,$04 ; 1,32$ \\
\hline \multicolumn{3}{|c|}{ Consumo de verduras ou legumes ${ }^{c}$} \\
\hline Inadequado & 1,00 & - \\
\hline Adequado & 1,33 & 1,$22 ; 1,45$ \\
\hline \multicolumn{3}{|c|}{ Consumo de frutas' } \\
\hline Inadequado & 1,00 & - \\
\hline Adequado & 1,35 & 1,$22 ; 1,48$ \\
\hline \multicolumn{3}{|c|}{ Consumo de peixe $(n=1.177)^{e}$} \\
\hline Inadequado & 1,00 & - \\
\hline Adequado & 1,15 & 1,$04 ; 1,26$ \\
\hline \multicolumn{3}{|c|}{ Consumo de refrigerante e sucos artificiais ${ }^{d}$} \\
\hline Inadequado & 1,00 & - \\
\hline Adequado & 1,15 & 1,$06 ; 1,24$ \\
\hline \multicolumn{3}{|c|}{ Consumo de alimentos doces $^{\mathrm{d}}(n=1.239)^{\mathrm{e}}$} \\
\hline Inadequado & 1,00 & - \\
\hline Adequado & 1,01 & 0,$93 ; 1,09$ \\
\hline \multicolumn{3}{|l|}{ Sal adicionald } \\
\hline Não & 1,00 & - \\
\hline Sim & 0,90 & 0,$76 ; 1,03$ \\
\hline \multicolumn{3}{|c|}{ Hábito de comer gordura aparente de carne vermelha e/ou pele de frango } \\
\hline Não & 1,00 & - \\
\hline Sim & 0,85 & 0,$77 ; 0,93$ \\
\hline
\end{tabular}

a) RP: razão de prevalência.

b) $\mathrm{IC}_{95 \%}$ : intervalo de confiança de $95 \%$.

c) Adequado $=$ quando relatado consumo de 3 dias ou mais por semana

d) Adequado = quando relatado consumo até 2 dias por semana.

e) Valor da amostra disponível para essa variável.

Nota: Foi considerado o efeito de delineamento amostral. 
Tabela 4 - Associação entre a autopercepção positiva da alimentação e indicadores de alimentação saudável, estratificada por sexo, em adultos e idosos ( $n=898$ ), Rio Grande, Rio Grande do Sul, 2016

\begin{tabular}{|c|c|c|c|}
\hline \multirow[b]{2}{*}{ Variáveis } & \multicolumn{3}{|c|}{ Ocorrência de autopercepção de alimentação positiva } \\
\hline & $\begin{array}{c}\text { Feminino } \\
(n=498) \\
n(\%)\end{array}$ & $\begin{array}{c}\text { Masculino } \\
(n=400) \\
n(\%)\end{array}$ & Valor $p^{a}$ \\
\hline Realização do café da manhã & $452(73,6)$ & $354(78,7)$ & 0,075 \\
\hline Realização do almoço & $493(71,8)$ & $398(74,3)$ & 0,402 \\
\hline Realização do lanche ou café da tarde & $416(72,5)$ & $297(77,8)$ & 0,068 \\
\hline Realização do jantar & $458(70,7)$ & $366(73,9)$ & 0,287 \\
\hline Realização do lanche ou café antes de dormir & $145(82,4)$ & $131(0,4)$ & 0,443 \\
\hline Consumo de leguminosas adequado ${ }^{b}$ & $387(73,6)$ & $332(76,5)$ & 0,319 \\
\hline Consumo de verduras ou legumes adequado ${ }^{b}$ & $360(79,7)$ & $248(79,7)$ & 1,000 \\
\hline Consumo de frutas adequado ${ }^{b}$ & $358(79,6)$ & $254(81,2)$ & 0,549 \\
\hline Consumo de peixe adequado ${ }^{b}$ & $33(82,5)$ & $37(82,2)$ & 0,906 \\
\hline Consumo de refrigerante e sucos artificiais adequadoc & $231(66,0)$ & $225(69,7)$ & 0,238 \\
\hline Consumo de alimentos doces adequadoc & $249(71,5)$ & $189(73,4)$ & 0,527 \\
\hline Não adiciona mais sal à comida & $454(71,2)$ & $362(75,7)$ & 0,130 \\
\hline Não consumo de gordura aparente de carne vermelha e/ou pele de frango & $182(63,4)$ & $206(68,2)$ & 0,133 \\
\hline
\end{tabular}

a) Valor-p obtido pelo teste de proporções.

b) Adequado = quando relatado consumo de 3 dias ou mais por semana

c) Adequado $=$ quando relatado consumo até 2 dias por semana.

\section{Discussão}

Entre os principais achados do presente estudo, destaca-se a prevalência de autopercepção positiva da alimentação e sua associação com dez indicadores de consumo alimentar adequado. Os indivíduos de mais idade, maior renda e considerados suficientemente ativos apresentaram maior frequência de autopercepção positiva de sua alimentação, contrariamente aos que referiram depressão e insegurança alimentar leve.

A prevalência de autopercepção positiva da alimentação, observada no presente estudo, é semelhante à encontrada em outras investigações realizadas no Brasil..$^{10,19} \mathrm{Em}$ 2016, um estudo desenvolvido na região Sul do país revelou uma prevalência de 69\% de autopercepção positiva da alimentação, em uma amostra de adultos e idosos usuários da rede urbana de atenção básica à saúde. ${ }^{10}$ No estudo conduzido pelo National Health and Nutrition Exame Survey (NHANES) 2005-2006, entretanto, somente 33\% da população adulta dos Estados Unidos perceberam sua dieta como excelente ou muito boa (escore alto) e $42 \%$ consideraram-na boa (escore médio). ${ }^{14} \mathrm{~A}$ prevalência da autopercepção positiva da alimentação observada nos Estados Unidos seria próxima à do presente estudo se, no estudo norte-americano, a classificação 'boa' (escore médio) tivesse sido considerada como positiva. Diferenças metodológicas, como o tipo de instrumento utilizado para medir o desfecho, podem ser observadas entre estudos, ressaltando a importância da definição dos critérios de avaliação no sentido de se permitir maior comparabilidade entre a literatura.

Diferenças na autopercepção positiva da alimentação podem ocorrer também por outros motivos, entre os quais se destacam os culturais (diferentes considerações do que é adequado, conforme países e culturas) e os temporais (diferentes épocas em que os estudos são realizados). Primeiramente, é importante definir uma alimentação adequada, de acordo com as recomendações do Ministério da Saúde - a exemplo do Guia Alimentar para a População Brasileira -, ${ }^{3}$ considerando-se a variabilidade do conceito ao longo do tempo, conforme os motivos anteriormente citados, e sua influência na percepção sobre o que cada pessoa considera adequado em termos da própria alimentação.

No presente estudo, dos 13 indicadores de alimentação saudável estudados, dez foram associados 
ao desfecho. A associação da autopercepção de alimentação com desfechos em saúde e de alimentação é relatada em outros estudos de destaque na literatura. ${ }^{14,19-21}$ Logo, a autopercepção de alimentação pode ser uma forma simples e viável de inferir a qualidade da alimentação do indivíduo. ${ }^{4,22}$

0 sexo feminino apresentou menor percepção alimentar positiva do que o sexo masculino, possivelmente explicada pelo fato de as mulheres demonstrarem mais atenção a aspectos de cuidados com a saúde e a alimentação e, sendo assim, terem uma melhor identificação do que é saudável e se mostrarem mais criteriosas em sua autoavaliação. ${ }^{10}$ Os homens, historicamente associados a piores cuidados com a saúde e uma alimentação com mais gorduras e maior ingestão de bebidas alcoólicas, possivelmente têm menor percepção do impacto desses hábitos sobre a manutenção de uma vida saudável. ${ }^{10,19}$

A idade se associou a uma melhor autopercepção da alimentação. Sabe-se que indivíduos de mais idade apresentam maior variedade no consumo dos alimentos. ${ }^{6,8,14,20}$ Os idosos também podem restringir o consumo de alguns alimentos, como medida preventiva e/ou terapêutica frente a doenças crônicas e, como consequência dessa atitude, considerar sua alimentação mais saudável. ${ }^{8,14}$ No presente estudo, a escolaridade não apresentou diferença estatística com a percepção da alimentação. Não obstante, outros estudos encontraram indivíduos com menor escolaridade a apresentar menor percepção alimentar, menor acesso à informação e ao cuidado em saúde. .3,24 $^{2}$

Quanto à renda e à segurança alimentar, ambas se associaram ao desfecho. Sabe-se que, entre os indivíduos mais pobres, o preço é um critério de escolha para a aquisição de produtos alimentícios e pode influenciar não só a diminuição da quantidade de alimentos, como também a perda de sua qualidade nutritiva e variedade, ${ }^{6,23}$ impactando de forma negativa o comportamento de consumo saudável. 5,6,21

Observou-se que indivíduos com pior percepção de seu estado de saúde tiveram uma redução da percepção positiva de sua alimentação, ao contrário dos indivíduos considerados ativos fisicamente, que tiveram uma melhor percepção em relação aos inativos. É consenso na literatura que indivíduos com melhor percepção de saúde são mais propensos a cuidar de sua saúde como um todo, apresentando comportamentos mais saudáveis. . $^{10,19,21,25,26}$
Entre os avaliados com depressão, verificou-se pior percepção da alimentação, o que reforça a importância da atenção à alimentação nesse grupo. Indivíduos deprimidos apresentam comportamentos não saudáveis como, por exemplo, má alimentação, ingestão alimentar compensatória de acordo com o presente estado de humor, sedentarismo e alterações no sono. ${ }^{14}$

0 estudo em tela apresenta algumas limitações. A primeira está no delineamento transversal, pois é possível que indivíduos com autopercepção positiva da alimentação tenham realizado mudanças recentes em seus hábitos alimentares, e a alimentação saudável referida seja um hábito mais recente, não de longa data. Tampouco se descarta outros tipos de viés, como aqueles relacionados ao consumo alimentar e à possibilidade de os indivíduos responderem o que acreditam ser mais adequado, ou ainda, socialmente aceito, sobretudo quando questionados por outra pessoa. Entretanto, os itens de consumo alimentar foram associados de forma significativa a perguntas de autopercepção positiva, sugerindo que os indivíduos foram consistentes em suas respostas. 0 delineamento transversal é útil na identificação de grupos-alvo, como, por exemplo, indivíduos que pretendam ou se sintam no dever de melhorar sua alimentação, ou com morbidades já instaladas - diabetes, hipertensão, hipercolesterolêmia -, necessitados de ações efetivas e específicas. Outra limitação refere-se ao uso de questões subjetivas, cujas respostas são condicionadas à interpretação do indivíduo sobre o que é questionado.

Entre os pontos fortes do estudo, destaca-se o delineamento de base populacional e o baixo número de perdas e recusas, reduzindo-se a probabilidade de viés de seleção, além do treinamento dos entrevistadores e do controle de qualidade aplicado.

Como conclusão, os achados mostraram que a autopercepção positiva de uma alimentação se relacionou com dez dos 13 indicadores alimentares avaliados. 0 autodiagnóstico negativo da alimentação pode sensibilizar e estimular a necessidade de mudanças comportamentais, assim como provocar o processo de adoção de hábitos alimentares adequados. Essa informação pode ser útil aos profissionais diretamente envolvidos com a orientação de saúde dos indivíduos. Contudo, mais estudos sobre o tema são necessários para melhor compreender como a autopercepção se reflete nas práticas alimentares. 


\section{Contribuição dos autores}

Machado KP participou do delineamento do estudo e do trabalho de campo, analisou os dados coletados, interpretou os resultados e foi responsável pela redação do manuscrito. Vaz JS contribuiu nas análises dos dados, redação e revisão do manuscrito.

\section{Referências}

1. Toral N, Slater B, Cintra IP, Fisberg M. Adolescent eating behavior regarding fruit and vegetable intakes. Rev Nutr [Internet]. 2006 May-Jun [cited 2019 Jun 19];19(3):331-40. Available from: http://www.scielo. br/pdf/rn/v19n3/30138.pdf. doi: 10.1590/\$141552732006000300004

2. Gregory CA, Smith TA, Wendt M. How Americans rate their diet quality: an increasingly realistic perspective. Econ Inf Bul [Internet]. 2011 Sep [cited 2019 Jun 19];83:1-18. Available from: https://www.ers.usda.gov/ webdocs/publications/44590/6042_eib83_1_.pdf?v=0

3. Ministério da Saúde (BR). Secretaria de Atenção à Saúde. Departamento de Atenção Básica. Coordenação Geral da Política de Alimentação e Nutrição. Guia alimentar para a população brasileira. 2. ed. Brasília: Ministério da Saúde; 2014 [citado 2019 jun 19]. 156 p. Disponível em: http://bvsms. saude.gov.br/bvs/publicacoes/guia_alimentar_ populacao_brasileira_2ed.pdf

4. Rodrigues PRM, Gonçalves-Silva RMV, Ferreira MG, Pereira RA. Viabilidade do uso de uma questão simplificada na avaliação da qualidade da dieta de adolescentes. Ciênc Saúde Coletiva [Internet]. 2017 maio [citado 2019 jun 19];22(5):156578. Disponível em: http://www.scielo.br/pdf/ csc/v22n5/1413-8123-csc-22-05-1565.pdf. doi: 10.1590/1413-81232017225.14102015

5. Strachan S, Brawley L. Healthy-eater identity and selfefficacy predict healthy eating behavior: a prospective view. J Health Psychol [Internet]. 2009 Jul [cited 2019 Jun 19];14(5):684-95. Available from: https://journals. sagepub.com/doi/abs/10.1177/1359105309104915. doi: $10.1177 / 1359105309104915$

6. Defante LR, Nascimento LDO, Lima-Filho DO. Comportamento de consumo de alimentos de famílias de baixa renda de pequenas cidades brasileiras: 0 caso de Mato Grosso do Sul. Interações (Campo Grande) [Internet]. 2015 jul-dez [citado 2019 jun 19];16(2):265-76. Disponível em: http://www.scielo. br/pdf/inter/v16n2/1518-7012-inter-16-02-0265.pdf. doi: $10.1590 / 151870122015203$
Mendonza-Sassi RA contribuiu com a supervisão do trabalho, desde sua concepção até sua finalização, revisou as análises dos dados, participou na interpretação de resultados e revisão crítica do conteúdo do manuscrito. Todos os autores aprovaram a versão final e são responsáveis por todos os aspectos do trabalho, incluindo a garantia de sua precisão e integridade.

7. Cade J, Thompson R, Burley V, Warm D. Development, validation and utilisation of food-frequency questionnaires - a review. Public Health Nutr [Internet]. 2002 Aug [cited 2019 Jun 19];5(4):56787. Available from: https://www.cambridge.org/core/ journals/public-health-nutrition/article/developmentvalidation-and-utilisation-of-foodfrequencyquestionnaires-a-review/463EFE9970053E8BD922CC 88F52E6244. doi: 10.1079/PHN2001318

8. Henn RL, Fuchs SC, Moreira LB, Fuchs FD. Development and validation of a food frequency questionnaire (FFQ-Porto Alegre) for adolescent, adult and elderly populations from Southern Brazil. Cad Saúde Pública [Internet]. 2010 nov [citado 2019 jun 19];26(11):2068-79. Disponível em: http:// www.scielo.br/pdf/csp/v26n11/08.pdf. doi: 10.1590/ S0102-311X2010001100008

9. Gomes AA, Pereira RA, Yokoo EM. Characterization of adults food intake based on simplified questionnaire: contribution to food and nutrition surveillance studies. Cad Saúde Colet [Internet]. 2015 Oct-Dec [cited 2019 Jun 19];23(4):368-73. Available from: http://www. scielo.br/pdf/cadsc/223n4/1414-462X-cadsc-23-4-368. pdf. doi: 10.1590/1414-462X201500040055

10. Lindemann IL, Oliveira RR, Mendoza-Sassi RA. Difficulties in ensuring a healthy diet among primary health care users and associated factors. Ciênc Saúde Coletiva [Internet]. 2016 Feb [cited 2019 Jun 19];21(2):599-610. Available from: http://www.scielo. br/pdf/csc/v21n2/1413-8123-csc-21-02-0599.pdf. doi: 10.1590/1413-81232015212.04262015

11. Dumith SC, Paulitsch RG, Carpena MX, Muraro MFR, Simões MO, Machado KP, et al. Planejamento e execução de um inquérito populacional de saúde por meio de consórcio de pesquisa multidisciplinar. Sci Med [Internet]. 2018 Jul-Sep [cited 2019 Jun 19];28(3):1-8. Available from: http:// revistaseletronicas.pucrs.br/scientiamedica/ojs/index. php/scientiamedica/article/view/30407

12. Instituto Brasileiro de Geografa e Estatistica. Censo demográfico 2010 [Internet]. Rio de Janeiro: Instituto 
Brasileiro de Geografa e Estatistica; 2011 [cited 2019 Jun 19]. Available from: https://ww2.ibge.gov.br/ home/estatistica/populacao/censo2010/default.shtm

13. Instituto Brasileiro de Geografia e Estatística. Pesquisa nacional por amostras de domicílios 2013 [Internet]. Rio de Janeiro: Instituto Brasileiro de Geografia e Estatística; 2014 [citado 2019 jun 19]. Disponível em: https://ww2.ibge.gov.br/home/estatistica/populacao/ trabalhoerendimento/pnad2013/default.shtm

14. Powell-Wiley TM, Miller PE, Agyemang P, AgursCollins T, Reedy J. Perceived and objective diet quality in US adults: a cross-sectional analysis of the National Health and Nutrition Examination Survey (NHANES). Public Health Nutr [Internet]. 2014 Dec [cited 2019 Jun 19];17(12):2641-9. Available from: https://www. ncbi.nlm.nih.gov/pmc/articles/PMC4190093/. doi: $10.1017 / \mathrm{S} 1368980014000196$

15. Matsudo S, Araujo T, Matsudo V, Andrade D, Andrade E, Oliveira LC, et al. Questionário internacional de atividade fisica (IPAQ): estudo de validade e reprodutibilidade no Brasil [Internet]. Rev Bras Ativ Fís Saúde [Internet]. 2001 dez [citado 2019 jun 19];6(2):5-18. Disponível em: http://rbafs.org.br/RBAFS/article/view/931/1222. doi: 10.12820/rbafs.v.6n2p5-18

16. Santos IS, Tavares BF, Munhoz TN, Almeida LSP, Silva NTB, Tams BD, et al. Sensibilidade e especificidade do Patient Health Questionnaire-9 (PHQ-9) entre adultos da população geral. Cad Saúde Pública [Internet]. 2013 Aug [cited 2019 Jun 19];29(8):1533-43. Available from: http://www.scielo.br/pdf/csp/v29n8/ v29n8a06.pdf. doi: 10.1590/0102-311X00144612

17. Pérez-Escamilla R, Segall-Corrêa AM, Maranha LK, Sampaio MFA, Marín-León L, Panigassi G. An adapted version of the U.S. Department of Agriculture Food Insecurity Module is a valid tool for assessing household food insecurity in Campinas, Brazil. J Nutr [Internet]. 2004 Aug [cited 2019 Jun 19];134(8):1923-8. Available from: https://academic. oup.com/jn/article/134/8/1923/4688851. doi: $10.1093 / \mathrm{jn} / 134.8 .1923$

18. Landis JR, Koch GG. The measurement of observer agrément for categorical data. Biometrics [Internet]. 1977 Mar [cited 2019 Jun 19];33(1):159-

74. Available from: https://www.jstor.org/ stable/2529310?seq=1\#page_scan_tab_contents. doi: $10.2307 / 2529310$

19. Jaime PC, Stopa SR, Oliveira TP, Vieira ML, Szwarcwald CL, Malta DC. Prevalência e distribuição sociodemográfica de marcadores de alimentação saudável, Pesquisa Nacional de Saúde, Brasil 2013. Epidemiol Serv Saúde [Internet]. 2015 abr-jun [citado
2019 jun 19];24(2):267-76. Disponível em: http://www. scielo.br/pdf/ress/v24n2/2237-9622-ress-24-02-00267. pdf. doi: 10.5123/\$1679-49742015000200009

20. Kye SY, Yun EH, Park KH. Factors related to selfperception of diet quality among South Korean adults. Asian Pac J Cancer Prev [Internet]. 2012 Apr [cited 2019 Jun 19];13(4):1495-504. Available from: http:// 13.228.5.104/?sid=Entrez:PubMed\&id=pmid:227993 $55 \& \mathrm{key}=2012.13 .4 .1495$

21. Reichert FF, Loch MR, Capilheira MF. Self-reported health status in adolescents, adults and the elderly. Ciên Saúde Colet [Internet]. 2012 Dec [cited 2019 Jun 19];17(12):3353-62. Available from: http://www. scielo.br/pdf/csc/v17n12/20.pdf. doi: 10.1590/\$141381232012001200020

22. Santos JV, Gigante DP, Domingues MR. Prevalence of food insecurity in Pelotas, Rio Grande do Sul State, Brazil, and associated nutritional status. Cad Saúde Pública [Internet]. 2010 Jan [cited 2019 Jun 19];26(1):41-9. Available from: http://www.scielo. br/pdf/csp/v26n1/05.pdf. doi: 10.1590/S0102311X2010000100005

23. Lins APM, Sichieri R, Coutinho WF, Ramos EG, Peixoto MVM, Fonseca VM. Alimentação saudável, escolaridade e excesso de peso entre mulheres de baixa renda. Ciênc Saúde Coletiva [Internet]. 2013 fev [citado 2019 jun 19];18 (2):357-66. Disponível em: http://www.scielo.br/pdf/csc/v18n2/07.pdf. doi: 10.1590/\$1413-81232013000200007

24. DeSalvo KB, Bloser N, Reynolds K, He J, Muntner P. Mortality prediction with a single general self-rated health question a meta-analysis. J Gen Intern Med [Internet]. 2006 Mar [cited 2019 Jun 19];21(3):26775. Available from: https://www.ncbi.nlm.nih.gov/ pmc/articles/PMC1828094/. doi: 10.1111/j.15251497.2005.00291.x

25. Sundquist J, Johansson SE. Self reported poor health and low educational level predictors for mortality: a population based follow up study of 39156 people in Sweden. J Epidemiol Community Health [Internet]. 1997 Feb [cited 2019 Jun 19];51(1):35-40. Available from: https://jech.bmj.com/content/51/1/35.long

26. Agostinho MR, Oliveira MC, Pinto MEB, Balardin GU, Harzheim E. Self-perceived health of adults users of Primary Health Care Services in Porto Alegre, RS, Brazil. Rev Bras Med Fam Comunidade [Internet]. 2010 Jan-Dec [cited 2019 Jun 19];5(17):9-15. Avaialble from: https:/www.researchgate.net/ publication/307646105_Self-perceived_health_of_ adults_users_of_Primary_Health_Care_Services_in_ Porto_Alegre_RS_Brazil 


\section{Abstract}

Objective: to analyze prevalence of positive selfperception of diet and association with sociodemographic and behavioral factors, morbidity, self-perception of health/weight, and body weight care, in the city of Rio Grande, RS, Brazil, in 2016. Methods: this was a crosssectional study conducted with adults and elderly people resident in the city of Rio Grande; 13 indicators of healthy eating and positive self-perception of diet (outcome) were applied; Poisson regression was used. Results: the sample consisted of 1,243 individuals, $72.4 \%$ of whom reported positive self-perception of diet; indicators such as weekly consumption of greens or vegetables $(P R=133-95 \% C I 1.22 ; 1.45)$ and fruit $(P R=1.35-95 \% C I$ 1.22;1.48) were positively associated with the outcome; no significant difference was found between sexes. Conclusion: positive self-perception of diet is associated with healthy eating indicators among adults and the elderly and can be useful in epidemiological surveys.

Keywords: Food Consumption; Self Report; Health Surveys; Cross-Sectional Studies.

\section{Resumen}

Objetivo: investigar la prevalencia de autopercepción positiva de la alimentación, sus factores sociodemográficos y comportamentales, morbilidades, autopercepción de la salud/peso y cuidados con el peso corporal en el municipio de Rio Grande, RS, Brasil, en 2016. Métodos: estudio transversal realizado con adultos y ancianos residentes en el municipio de Rio Grande; se aplicaron 13 indicadores de alimentación saludable y autopercepción de la alimentación; se utilizó la regresión de Poisson. Resultados: la muestra fue de 1.243 individuos, $72,4 \%$ refirió autopercepción positiva de la alimentación; indicadores como consumo semanal de verduras o legumbres $\left(R P=1,33-I C_{95 \%} 1,22 ; 1,45\right)$ y frutas $(R P=1,35$ - IC $\left.C_{95 \%} 1,22 ; 1,48\right)$ fueron positivamente asociadas al desenlace ( $p<0,001)$; no bubo diferencia significativa entre los sexos. Conclusión: la autopercepción positiva de la alimentación se asocia con indicadores de alimentación entre adultos y ancianos pudiendo ser útil en estudios epidemiológicos.

Palabras-clave: Consumo de Alimentos; Autoinforme; Encuestas Epidemiológicas; Estudios Transversales.

Recebido em 06/09/2018

Aprovado em 11/05/2019 